\title{
300kV Performance of a 10k x 10k Scintillator- and Fiber-Coupled CCD Camera for Transmission Electron Microscopes
}

\author{
B. C. Lee, ${ }^{*}$ B. Mollon, * P. E. Mooney* \\ * Gatan, Inc., 5794 W. Las Positas Blvd., Pleasanton CA 94588
}

Gatan, Inc. recently introduced the UltraScan 10000 XP / model 990 10k x 10k CCD camera for TEMs. We present here $300 \mathrm{kV}$ resolution results based on data from the camera recently installed at the National Center for Macromolecular Imaging (NCMI), Baylor College of Medicine, Houston, Texas (Fig. 1). We compare this with data gathered from NCMI's UltraScan $40004 \mathrm{k} x$ 4k camera, characterized in [1] and [2].

The US 10000 XP camera sensor is a $10560 \times 10560$ pixel (111 megapixel) CCD - the largest CCD currently produced - with $100 \%$ fill factor 9um pixels for a total imaging area of $95 \mathrm{~mm} \times 95 \mathrm{~mm}$. This represents 2.4 times the imaging area of the US 4000 and over 6.6 times the pixel count. The camera provides binning modes of $1 \mathrm{x}, 2 \mathrm{x}, 3 \mathrm{x}, 4 \mathrm{x}, 5 \mathrm{x}, 10 \mathrm{x}$ and $20 \mathrm{x}$ in low noise $(\approx 10 \mathrm{CCD}$ electron) $1 \mathrm{MHz}$ readout mode, and faster $10 \mathrm{MHz}$ readout for $1 \mathrm{x}$ and $2 \mathrm{x}$ binning. With 16 port readout at $10 \mathrm{MHz}$ the entire 10k x 10k CCD image can be read in less than 1 second. The 20x binning mode provides a $180 \mu \mathrm{m}$ high sensitivity low crosstalk pixel with 10 frames per second readout, and the same low noise as $1 \mathrm{x}$ binning. The CCD is coupled to Gatan's next-generation high-contrast resolution $(\mathrm{HCR}+\mathrm{TM})$ fiber optics and utilizes a newly developed high resolution scintillator in order to improve system resolution and take advantage of the higher density $9 \mu \mathrm{m}$ CCD pixels (compared to the $15 \mu \mathrm{m}$ pixel UltraScan $40004 \mathrm{k}$ x 4k camera). The NCMI camera scintillator is optimized for low dose single particle applications, providing a balance of sensitivity and resolution.

DQE was measured using methods outlined in [3] and [4], based on ISO12233 as described in [5], using a tilted edge from the TEM beamstop (Fig. 2). DQE results at $300 \mathrm{kV}$ show substantially improved resolution on an absolute (line pairs per $\mathrm{mm}$ ) scale for the $10 \mathrm{k} \mathrm{x} 10 \mathrm{k}$ camera over the $4 \mathrm{k} \mathrm{x}$ 4k camera (Fig. 3), demonstrating the improved optics and the improvement from finer sampling. The increase in DQE brings the 10k x 10k camera to near pixel equivalence with the larger US 4000 pixels at $300 \mathrm{kV}$, such that the collected data scales closer to pixel count than physical area. Therefore, at the same dose level per pixel this detector is expected to deliver approximately 6 times the information content per image as a typical $4 \mathrm{k}$ x $4 \mathrm{k}$ scintillator- and fiber-coupled CCD camera.

References

[1] C. R. Booth et al., Journal of Structural Biology. 156 (2006) 556.

[2] D.-H. Chen et al., Journal of Structural Biology. 163 (2008) 45.

[3] P. Mooney, Methods Cell Biol. 79 (2007) 661.

[4] P. Mooney, Microsc. Microanal. 15 (Suppl. 2) (2009) 234.

[5] E. Samei et al., Med Phys. 25 (1998) 102. 

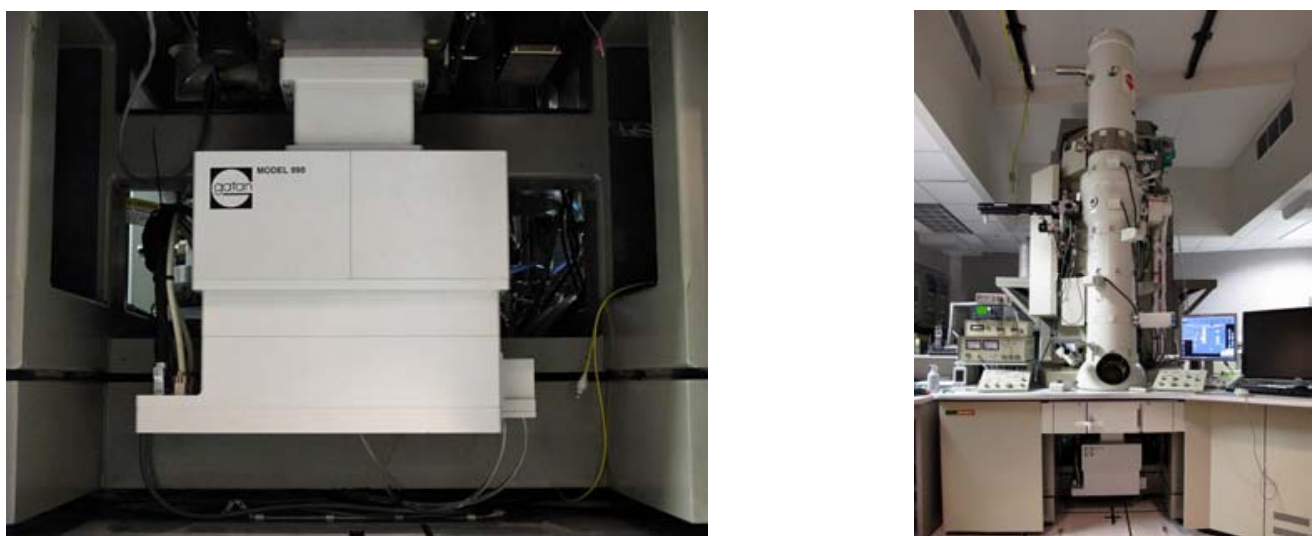

FIG. 1. US $10000 \mathrm{XP} /$ model 990 10k x 10k camera installed on a JEOL 3200 FSC TEM at the National Center for Macromolecular Imaging (NCMI), Baylor College of Medicine, Houston, Texas. The camera fits within the unmodified kneewell shield (removed in these photos).

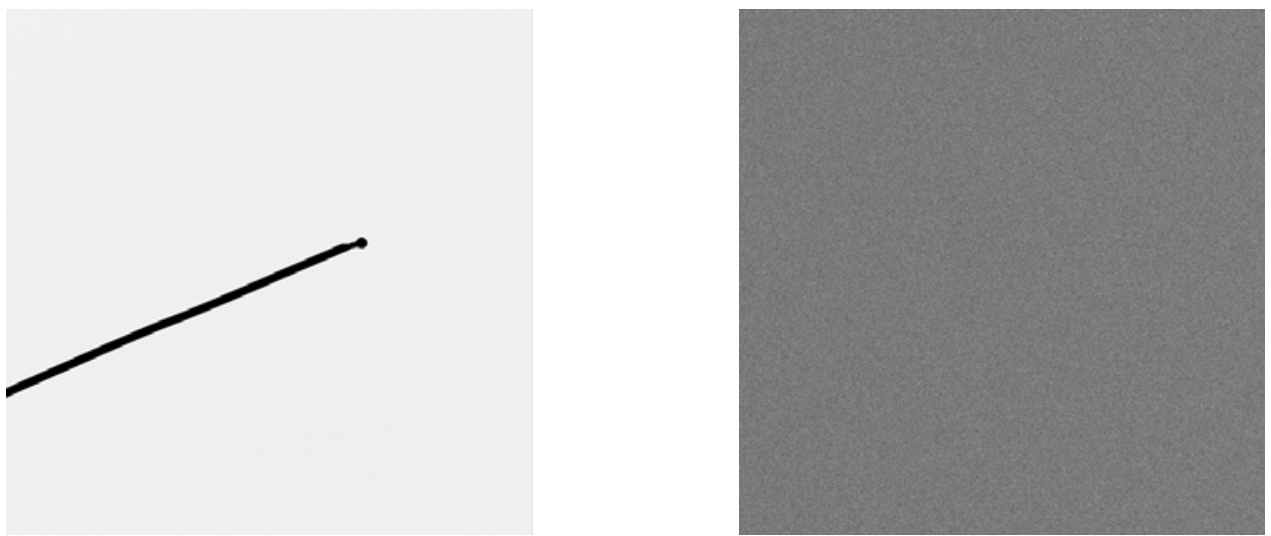

FIG. 2. $10 \mathrm{k} \times 10 \mathrm{k} 1 \mathrm{MHz}$ mode DQE test images at $300 \mathrm{kV}$. On the left is a beam stop image, the slanted edge is used to determine MTF. On the right is a uniform image with automatic contrast stretch, showing no trace of the 16 separate readout sections.
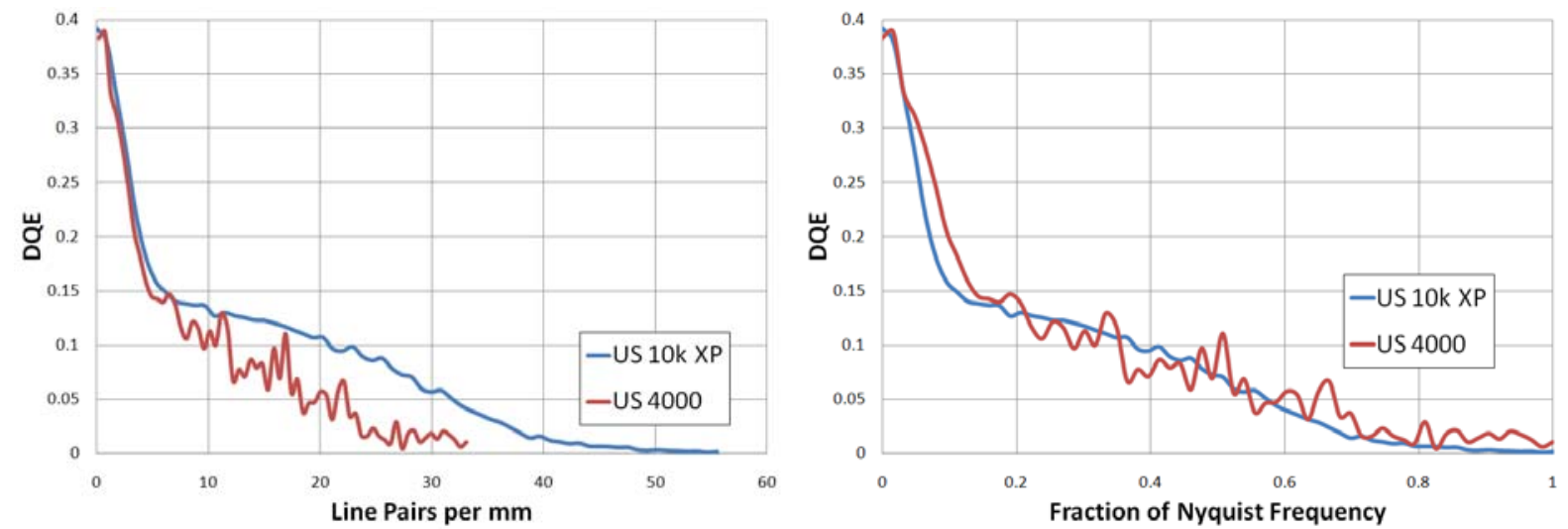

FIG. 3. DQE at $300 \mathrm{kV}$ for the US $10000 \mathrm{XP} 10 \mathrm{k} \times 10 \mathrm{k}$ camera and the US $40004 \mathrm{k} \times 4 \mathrm{k}$ camera; the plot on the left is a function of physical dimensions where frequency is in line pairs per $\mathrm{mm}$. On the right, DQE is plotted as a function of each camera's Nyquist Frequency, determined by the CCD pixel size. The US $10000 \mathrm{XP}$ with $9 \mu \mathrm{m}$ pixels shows similar per pixel performance to the US 4000 with larger $15 \mu \mathrm{m}$ pixels. 